Erschienen in: Gunkel, Lutz/Zifonun, Gisela (Hrsg.): Deutsch im

Sprachvergleich. Grammatische Kontraste und Konvergenzen. - Berlin,

Boston: de Gruyter, 2012. S. 217-238. (Jahrbuch des Instituts für Deutsche

Sprache 2011), https://doi.org/10.1515/9783110284768.217

Patricia Cabredo Hofherr

\title{
Verschmelzungsformen von Präposition und Artikel. Deutsch und Französisch kontrastiv ${ }^{1}$
}

\begin{abstract}
Die genaue Charakterisierung der möglichen Wechselwirkungen zwischen Syntax und Morphologie stellt eine der zentralen Forschungsfragen der Sprachwissenschaft dar. Die hier betrachteten Verschmelzungsformen bieten sich als Fallstudie für die Syntax-Morphologie-Schnittstelle an, da Verschmelzungsformen von Präposition und Artikel wie $d u / a u$ im Französischen oder am/zum im Deutschen paradigmatisch Sequenzen gegenüberstehen, in denen eine nicht-reduzierte Präposition mit einem vollen Artikel kombiniert wird (de la/à lat bzw. an dem/ zu dem). Für die Analyse dieser Formen muss also untersucht werden, inwiefern die Verwendung von Verschmelzungsformen gegenüber unreduzierten Abfolgen Änderungen in der Syntax nach sich zieht. In diesem Beitrag werde ich zeigen, dass die Wechselbeziehungen zwischen Verschmelzungsform und Syntax im Französischen und Deutschen unterschiedlicher Natur sind. Französische und deutsche Verschmelzungs formen unterscheiden sich in ihren morphologischen, semantischen und syntaktischen Eigenschaften. Hier sollen zwei Eigenschaften genauer untersucht werden: (i) die Kombinierbarkeit von Verschmelzungsformen und Nominalphrasen mit restriktiven Relativsätzen im Deutschen ( $\mathrm{im} / \mathrm{in}$ dem Haus, das gerade renoviert nird), und (ii) die Koordinationsmöglichkeiten von Präpositionalphrasen mit Verschmelzungs formen im Französischen und im Deutschen. Es ist bekannt, dass Verschmelzungsformen im Französischen die Koordinationsmöglichkeiten der beteiligten Nominalphrasen einschränken. Vergleichbare Wechselwirkungen zwischen Verschmelzungsform und Koordination sind im Deutschen jedoch nicht zu beobachten, wie anhand von Koordinationsdaten aus dem COSMAS II-Korpus belegt werden kann.
\end{abstract}

\section{Einleitung}

Syntax und Morphologie stellen zwei Ebenen der grammatischen Struktur dar. Nun stellt sich die Frage, welche Wechselwirkungen zwischen syntaktischen Strukturen und den morphologischen Einheiten, die in ihnen verwendet werden, bestehen. Da die Unabhängigkeit von Syntax und Phonologie als methodologisches Prinzip wünschenswert ist, wie im , Principle of Phonology-Free Syntax ${ }^{6}$ von Zwicky/Pullum (1986) formuliert, muss für Fälle, in denen morphologische Struktur in Abhängigkeit von Merkmalskombinationen variiert, untersucht werden, inwiefern morphologisch be-

Ich möchte mich bei zwei anonymen Gutachtern für Vorschäge, Literaturverweise und Kommentare bedanken. Für ihre Hilfe mit den französischen Beispielen danke ich Olivier Bonami, Gilles Boyé und Sabrina Bendjaballah. Gerhard Schaden bin ich für die Beispiele aus dem Vorarlberger Dialekt dankbar. 
dingte phonologische Veränderungen syntaktische Veränderungen nach sich ziehen. Hier soll konkret der Frage nachgegangen werden, inwiefern die Verteilung von morphologischer Information über eine oder mehrere lexikalische Einheiten Einfluss auf die Syntax ausübt. Zwei Phänomene zeichnen sich durch Abweichungen vom erwarteten Parallelismus zwischen Syntax und Morphologie aus: Periphrasen zum einen und Verschmelzungsformen zum anderen.

Bei Periphrasen wird ein morphologisches Merkmalsbündel, das für andere Merkmalswerte in einer lexikalischen Einheit ausgedrückt wird, durch zwei lexikalische Einheiten realisiert (Ackema/Neeleman 2004, 2007; Börjars et al. 1997; Bresnan 2001; Chumakina/Corbett (Hg.) im Erscheinen; Embick 2007a; Embick/Noyer 2001, 2007; Hankamer/Mikkelsen 2005; Poser 1992; Stewart/Stump 2007).
a. il courait
er lief. IPFV ${ }^{2}$
b. il a couru
er ist gelaufen

Bei Verschmelzungsformen werden umgekehrt Merkmale, die für andere Merkmalskombinationen auf zwei kategoriell unterschiedliche lexikalische Einheiten verteilt sind, durch eine lexikalische Einheit dargestellt: ${ }^{3}$
a. die Reise zum Mond
b. die Reise zu den Seychellen

Die folgenden Überlegungen beschäftigen sich ausschließlich mit Verschmelzungsformen. Es soll ermittelt werden, ob für Verschmelzungsformen eine Wechselwirkung zwischen Morphologie und Syntax zu beobachten ist. Anders formuliert soll der Frage nachgegangen werden, ob Strukturen, in

2 In den Glossen werden folgende Abkürzungen verwendet: A = à , nach, zu'; DE = de, von'; IPFV = imperfektive Vergangenheit; LE = le (voller definiter Artikel, maskulin Singular); DET.RED = phonologisch reduzierter definiter Artikel $(l)$; DET $=$ definiter Artikel; $\mathrm{M}=$ Maskulinum; F = Femininum; $N=$ Neutrum; PL = Plural; $S G=$ Singular; $A C C=$ Akkusativ; DAT $=$ Dativ $;$ GEN $=$ Genitiv; NOM $=$ Nominativ .

3 Ein anonymer Gutachter weist berechtigterweise darauf hin, dass die Analyse als Periphrase oder als Verschmelzung davon abhängt, ob das Auftreten von zwei lexikalischen Einheiten als markiert oder als unmarkiert angesehen wird. In typischen Periphrasen - wie beispielsweise den verbalen Periphrasen der romanischen Sprachen - liegt ein Paradigma vor, dessen Zellen zum Großteil durch flektierte Formen des Grundlexems besetzt sind. In diesen Fällen wird die flektierte Form als Normalfall angesehen und die Realisierung einer Merkmalskombination von Tempus und Aspekt durch zwei lexikalische Einheiten wird als abweichende, periphrastische Realisierung eingestuft. In Fällen von Verschmelzung - wie beispielsweise den P+D-Verschmelzungen - tritt eine lexikalische Einheit in Kontexten auf, wo auforund der im Allgemeinfall festgestellten syntaktischen Struktur zwei syntaktische Endstellen erwartet werden. 
denen Verschmelzungsformen auftreten, sich syntaktisch von Strukturen mit Abfolgen nicht-reduzierter Formen von Präposition und Artikel unterscheiden.

\section{Eingrenzung der betrachteten Verschmelzungsformen}

Sowohl für das Deutsche als auch für das Französische werden im Folgenden ausschließlich Verschmelzungen von Präposition und Artikel untersucht. Diese rein kategorielle Definition muss jedoch weiter verfeinert werden, da die unterschiedlichen Verschmelzungen von Präposition und Artikel aufgrund ihrer syntaktischen und semantischen Eigenschaften weder im Französischen noch im Deutschen als homogene Klassen angesehen werden können. Die Eingrenzung der betrachteten Verschmelzungsformen muss also gerechtfertigt werden.

Für das Französische sollen hier nur die Verschmelzungen der Präpositionen $\grave{a}, \mathrm{zu}^{\prime}$ und $d e$, von' mit den Formen $a u / d u, z u+D E T . M S G / v o n+D E T$. $\mathrm{MS}^{6}$ und $a u x / d e s, z u+D E T$.PL/von+DET.PL' betrachtet werden. Diese Verschmelzungen treten an die Stelle der Abfolgen *àle $/ *$ de le sowie $* \dot{a}$ les $/ *$ de les:
a. le père
du/
de le
garçon
der Vater
$\mathrm{DE}+\mathrm{LE} /$
DE LE Junge
,der Vater des Jungenc
b. Elle parle au/ $*$ à le garçon. sie spricht A+LE/ A LE Junge ,Sie spricht mit dem Jungen.

Die Präpositionen à und de bilden mit der Präposition en in' die Klasse der schwachen Präpositionen des Französischen. Des Weiteren steht en ebenfalls mit einem Verschmelzungsphänomen in Verbindung, in dem eine erwartete Abfolge von Präposition und Artikel durch einen einzelnen lexikalischen Ausdruck ersetzt wird (siehe Miller/Pullum/Zwicky 1997, S. 81-82).

$$
\begin{aligned}
& \text { Il va } \\
& \text { er fährt }
\end{aligned}
$$
a. en France
b. en Amérique EN Frankreich *à la
France.
A DET.FSG Frankreich EN Amerika *à $\mathrm{l}^{\prime}$ Amérique
A DET.RED- Amerika.FSG
c. ${ }^{*}$ en Philippines
EN Philippinen
aux
Philippines
A+DET.PL
Philippinen
d. *en Indes
aux
Indes
EN Indien.pl
A+DET.PL
Indien.PL 


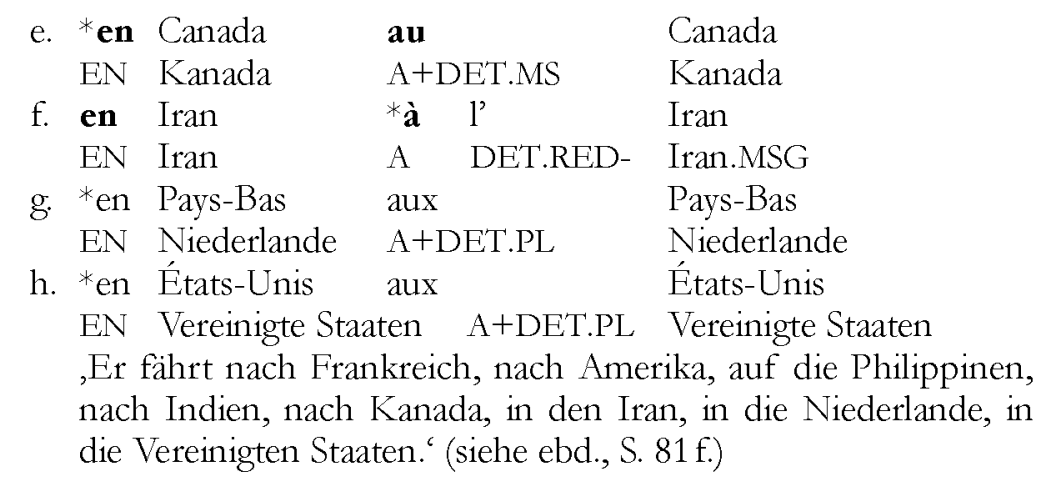

Die Verschmelzung en, die in diesen Beispielen die Abfolge à la und à l' ersetzt, unterscheidet sich jedoch eindeutig von den Verschmelzungen au/du/ aux/des.

Der erste Unterschied betrifft die Verteilung der Verschmelzungsformen. Die Verschmelzung mit en in (4) unterliegt zwei Einschränkungen: (1) der betroffene Artikel muss zu einem Eigennamen gehören und (ii) das PPKomplement muss lokativ gebraucht werden (Miller/Pullum/Zwicky 1997). Im Gegensatz dazu müssen die Verschmelzungen au/du/aux/des ungeachtet der semantischen Eigenschaften der Präpositionalphrase oder des Kopfnomens in allen Kontexten benutzt werden, in denen sonst ein Artikel le oder les auf die Präpositionen à oder de folgen würde.

a. Lokative PP, aber kein Eigenname:

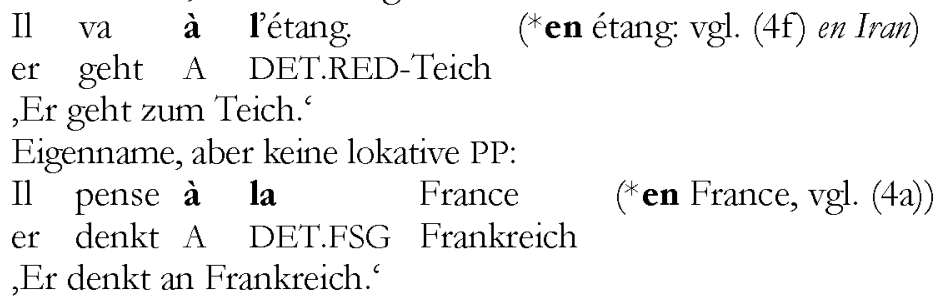

Der zweite Unterschied ist bei den Wechselwirkungen zwischen den Verschmelzungsformen und der Artikelreduktion (élision) zu beobachten: In den Fällen, in denen Artikelreduktion möglich wäre, erscheint die Verschmelzungsform en (siehe 4b/f) - die Verschmelzungsformen au/du können hingegen nie in Kontexten stehen, in denen Artikelreduktion erfolgen kann:
a. de l'oncle
$* \mathbf{d u}$
oncle
,DE+DET Onkel
b. à l'oncle
$*$ au oncle
,A+DET Onkel

Aufgrund dieser grundlegenden Unterschiede zwischen den Verschmelzungsformen wird die Verschmelzung en aus den folgenden Untersuchungen ausgeschlossen. 
Für das Deutsche sollen hier die folgenden Formen betrachtet werden:

\begin{tabular}{|l|l|l|l|l|l|}
\hline Genus/Kasus & an & bei & in & von & $\mathbf{z u}$ \\
\hline M.SG.ACC & - & $\mathrm{x}$ & - & $\mathbf{x}$ & $\mathbf{x}$ \\
\hline $\begin{array}{l}\text { M.SG.DAT } \\
(=\text { N.SG.DAT) }\end{array}$ & am & beim & im & vom & zum \\
\hline N.SG.ACC & ans & $\mathbf{x}$ & ins & $\mathbf{x}$ & $\mathbf{x}$ \\
\hline F.SG.ACC & - & $\mathbf{x}$ & - & $\mathbf{x}$ & $\mathbf{x}$ \\
\hline F.SG.DAT & - & - & - & - & zur \\
\hline
\end{tabular}

Diese Formen entsprechen im Wesentlichen dem im Duden (2006, S. 624) angeführten Inventar der Präpositionen, die besonders häufig in obligatorischen Verschmelzungen auftreten. Die folgenden Beispiele zeigen, dass für längere, weniger grammatikalisierte Präpositionen die Verschmelzungsformen nicht die gleichen Eigenschaften besitzen. Für die Verschmelzungen binterm und vorm in Verbindung mit Eigennamen (der Bodensee) wird beispielsweise im Gegensatz zu den anderen Verschmelzungen die kontrahierte Form nicht bevorzugt (7h-i) (siehe Wiegand 2000, S. 74 für ähnliche Beispiele).
a. Er wohnt am/*an dem Bodensee.
b. Er fährt ans $/{ }^{*}$ an das Mittelmeer.
c. Er wohnt beim $/{ }^{*}$ bei dem Bodensee.
d. Maria badet im/*in dem Bodensee.
e. Er fährt ins $/ *$ in das Rheinland.
f. Er fährt zum/* zu dem Bodensee.
g. Er fährt zur $/ *$ zu der Zugspitze.
h. Er stand vorm/vor dem Bodensee.
i. Er stand hinterm/hinter dem Dom.

Hier benutze ich die obligatorische Kontraktion mit Eigennamen als Ausschlusskriterium für Verschmelzungsformen. Wiegand (2000, S. 70, 76) verwendet die Interpretationsmöglichkeiten mit semantischen Unika im weiteren Sinn als Kriterium und stuft die Verschmelzungen ans und ins als Zwischenstufen ein, für die der Grammatikalisierungsprozess nicht abgeschlossen ist. Da hier gezeigt werden soll, dass die grammatikalisierten Verschmelzungen im Deutschen syntaktisch transparent bleiben, stützen sich die folgenden Überlegungen auf Belege mit den klar grammatikalisierten Formen am, beim, im, von, zum und qur. Vergleichbare Belege gibt es auch für ans und ins; diese sind jedoch weniger aussagekräftig, da bei weniger grammatikalisierten Formen ein höheres Maß an syntaktischer Transparenz erwartet wird. 


\section{Allgemeine Eigenschaften der Verschmelzungsformen}

Die Verschmelzungsformen $a u / d u / a u x / d e s$ heben sich in ihrer Verteilung klar von den Verschmelzungsformen des Deutschen ab: Im Französischen ist die Abfolge einer Präposition à de und eines bestimmten Artikels le oder les ungrammatisch und muss zwingend durch eine Verschmelzungsform aul du/ aux/des ersetzt werden (siehe Bsp. (3)). Im Gegensatz hierzu stehen allen Verschmelzungsformen des Deutschen die entsprechenden unverschmolzenen Abfolgen von Präposition und Artikel gegenüber (8).

a. Hans ist ins Kino gegangen.

b. Hans ist in das Kino gegangen.

Für die Kombinationen, die über eine Verschmelzungsform verfügen, ist eine semantische Differenzierung zwischen der Verschmelzungsform und der unverschmolzenen Abfolge zu beobachten. Wie Hartmann (1978, 1980, 1982) herausstellt, lässt sich der semantische Kontrast weitestgehend auf eine Differenzierung zwischen schwacher und starker Definitheit zurückführen. Hartmann weist darauf hin, dass die volle Abfolge von Präposition und Artikel für anaphorische und deiktische Lesarten verwendet wird, während Verschmelzungsformen für nicht-anaphorische und nicht-deiktische definite Lesarten (semantische Unika) Anwendung finden. Diese Unterscheidung entspricht der Unterscheidung zwischen semantischer und pragmatischer Definitheit (siehe Löbner 1985). ${ }^{4}$
a. Semantische Definitheit zum Mond am Bug/im Garten
b. Pragmatische Definitheit ${ }^{5}$ ein Auto (...) in dem Auto
(semantische Unika)
(situative Unika)
(anaphorisch)

Wie Wiegand (2000, S. 68f.) betont, trifft diese Behauptung nicht für alle verschmolzenen Formen des Deutschen gleichermaßen zu. Verschmelzungen mit einem geringen Grammatikalisierungsgrad - wie beispielsweise ïbers - lassen für die Verschmelzung und die unverschmolzene Abfolge spezifische und monosemantische Interpretationen zu. Komplementäre Distribution gilt für diese Verschmelzungen nur für generische und phorische Interpretation, wobei die Verschmelzung für eine generische Interpretation

${ }_{4} \quad$ Bei assoziativer Definitheit wird die Wahl des Artikels auch durch das semantische Verhältnis zwischen den Referenten bedingt. Wie Schwarz (2009) zeigt, wird bei assoziativer Definitheit, die durch Teil-Ganzes-Beziehungen legitimiert wird, der schwache Artikel bevorzugt; im Gegensatz hierzu wird bei assoziativer Definitheit zwischen Werk und Autor der starke Artikel vorgezogen.

5 Bei nominalen Anaphern (engl., epithets') muss ebenfalls der volle Artikel stehen.

(i) Herr Schmitt fing im Frühjahr 1940 in unserem Geschäft als Lehrling an. Uns allen war bald klar, dass meine Tante *am/an dem jungen Mann einen Narren gefressen hatte. 
verwendet werden muss und die Vollform für phorische Kontexte. Die hier betrachteten Verschmelzungsformen entprechen jedoch den speqiellen $P-A$-Verschmelzungen bei Wiegand, für die die semantische Differenzierung zutrifft. ${ }^{\circ}$

Der semantische Kontrast zwischen semantischer und pragmatischer Definitheit findet sich auch in Sprachen wieder, die zwei Arten definiter Artikel in ihrem Artikelsystem unterscheiden (Heinrich 1954 für den Dialekt von Amrum; Ebert 1971a, b für Fehring; Hartmann 1982 für den Dialekt von Mönchengladbach; Breu 2004 für das Obersorbische; Studler 2008 für eine Übersicht der schweizerdeutschen Dialekte). ${ }^{7}$

Im Folgenden soll der Frage nachgegangen werden, ob Verschmelzungsformen einen Einfluss auf die Syntax der sie enthaltenden Strukturen ausüben. Hierfür werden zwei unabhängige Eigenschaften von Verschmelzungsformen betrachtet: ihre Kombinierbarkeit mit restriktiven Relativsätzen (Abschnitt 4) und ihre Verteilung in Koordinationen von Präpositionalphrasen und Nominalphrasen (Abschnitt 5). Die Kombinierbarkeit mit restriktiven Relativsätzen beeinflusst nur im Deutschen die Verschmelzung von Präposition und Artikel, da im Französischen auch bei modifizierten Kopfnomen ausnahmslos eine Verschmelzungsform auftreten muss.

In der Argumentation werden als Belege Beispiele aus dem COSMAS IIKorpus verwendet, die zwei zusätzliche Auswahlkriterien erfüllen. Erstens wurden nur Beispieltypen berücksichtigt, die in Quellen unterschiedlichen geografischen Ursprungs erscheinen: Dies soll spezifische regionale Einflüsse ausschließen. Zweitens wurden nur Beispiele herangezogen, die von zusätzlich befragten Muttersprachlern als grammatisch bewertet wurden; hiermit soll vermieden werden, mögliche Kopierfehler als Belege einzuschließen.

\section{Verschmelzungsformen und Relativsätze: Syntax oder Semantik?}

In der Literatur wird im Allgemeinen die Auffassung vertreten, dass Verschmelzungsformen im Deutschen nicht mit Kopfnomen vereinbar sind, die durch restriktive Relativsätze modifiziert werden (Hartmann 1980, S. 172; Raffelsiefen 1987; Nübling 2005, S. 112; Duden 2006, S. 624; Puig-Waldmüller 2008, S. 148; Schwarz 2009, Kap. 2.1, 6.4.2). ${ }^{8}$

\footnotetext{
Abfolgen von Präposition und Artikel ohne entsprechende Verschmelzungsform decken ebenfalls sowohl die semantisch als auch die pragmatisch definiten Lesarten ab.

$7 \quad$ In den Arbeiten zu Sprachen mit zwei definiten Artikeln wird häufig ein sibwacher definiter Artikel von einem starken definiten Artikel unterschieden: Hierbei deckt der schwache Artikel im Allgemeinen die semantische Definitheit ab, während der starke Artikel pragmatische Definitheit kennzeichnet.

8 Eine abweichende Auffassung wird in Wiegand (2000) vertreten (siche Beispiele in Wiegand 2000, S. 72, 90); ich danke einem anonymen Gutachter für diesen Hinweis.
} 
(10) a. \#Im/In dem Haus, das verkauft wird, wohnt Fritz.

b. Sie geht \#zum/zu dem Zahnarzt, der ihr empfohlen wurde.

Hier soll untersucht werden, ob diese Einschränkung syntaktischer oder semantischer Natur ist. Eine grundsätzliche Unverträglichkeit zwischen Verschmelzungen und restriktiven Relativsätzen kann nicht angenommen werden, da es systematische Ausnahmen gibt, in denen Verschmelzungsformen mit einem restriktiven Relativsatz problemlos auftreten können (11). Wie die Beispiele (12) aus dem Vorarlberger Dialekt zeigen, trifft die entsprechende Beobachtung auch für schwache definite Artikel zu.

(11) a. Im Institut, in dem ich vorher arbeitete, war das kein Problem. (konstruiertes Beipiel)

b. Es dürfe nicht sein, dass Anwohner, die eine Nachtparkgebühr zahlen, im Wohnquartier, in dem sie leben, keinen freien Parkplatz mehr finden. (A97/SEP.22788 St. Galler Tagblatt, 08.09.1997)

c. Im Betrieb, in dem ich Werkzeugmacher gelernt habe, gab es 1000 Mitarbeiter. (BRZ08/APR.11354 Braunschweiger Zeitung, 22.04. 2008)

d. Der Besuch des ehemaligen Kellners im Privatclub, in dem sie arbeitete, endete für die Frau in Todesangst. (M98/DEZ.93016 Mannheimer Morgen, 09.12.1998)

e. Dass dieser „Platzkampf der deutschen Hirsche“, wie Dr. Elvira die sogenannte Zusammenarbeit dieser beiden Herren nannte, ausgerechnet nach Afrika getragen und im Gesundheitsprojekt, an dem Dr. Elvira beteiligt war, ausgetragen wurde, war eine peinliche Erfahrung für die Ärztin. (DIV/EEB.00001 Ellmer, Elfriede: Die Bettelfrau von Buhinga. Föritz, 2003, S. 136)

a. s/ *des inschtitut wo i frühr gschaffat ho $\mathrm{DET}_{\text {schwach }} / \mathrm{DET}_{\text {stark }}$ Institut wo ich früher gearbeitet habe isch bessr organisiert gsi.

ist besser organisiert gewesen.

b. mirhots/ des inschtitut wo $i$ frühr mirhat $\mathrm{DET}_{\text {schwach }} / \mathrm{DET}_{\text {stark }}$ Institut wo ich früher gschaffat ho bessr gfalla.? gearbeitet habe besser gefallen.

Wie der Kontrast zwischen den Beispielen (12a) und (12b) zeigt, trägt die Position der Nominalphrase im Vor- oder Mittelfeld zur Auswahl der Artikelform bei. Worauf dieser Kontrast letztendlich zurückzuführen ist, ist noch nicht klar. Für die Argumentation hier ist jedoch nur von Bedeutung, dass in beiden Fällen der schwache Artikel trotz des restriktiven Relativsatzes möglich ist. Ich danke Gerhard Schaden für diese Beispiele. 
Wie Ebert (1971, S. 169) zeigt, kann auch im Fehring unter bestimmten Bedingungen ein restriktiver Relativsatz mit einem schwachen Artikel am Kopfnomen kombiniert werden. Wespel (2008, S. 186) weist darauf hin, dass in dem von Ebert betrachteten Beispiel das Adjektiv iast, erstes ${ }^{c}$ eine Bedeutungsverschiebung verursacht: Das einfache Nomen buk, Buch' wird durch das Adjektiv zu einem Funktionalbegriff iast buk, erstes Buch`. Anders als ein einfaches Nomen weist ein Funktionalbegriff seinem Argument einen einzigen Referenten zu.

$\begin{array}{lllll}\text { a. Det/ *At buk, wat hi tuiast skrewen hee, } \\ \text { DET / DET } & \text { Buch was er zuerst geschrieben hat }\end{array}$ docht niks. taugt nichts.

b. Det/ At iast buk, wat hi skrewen hee, $\mathrm{DET}_{\text {stark }} / \mathrm{DET}_{\text {schwach }}$ erste Buch was er geschrieben hat docht niks. taugt nichts.

((33) und (33') in Ebert 1971b, S. 169 $)^{10}$

Wespels Beobachtung kann direkt auf die Beispiele mit Verschmelzungsformen in (11) übertragen werden: Restriktive Relativsätze sind mit Verschmelzungsformen an funktionalen Kopfnomen vereinbar. In diesen Fällen vervollständigt der Relativsatz eine funktionale Beschreibung, die in dem gegebenen Kontext auf einen Referenten verweist, der aufgrund der Semantik des Kopfnomens notwendigerweise eindeutig ist (siehe Bsp. 13).

Die hier betrachteten Beispiele mit funktionalen Kopfnomen stellen jedoch nicht die einzigen Fälle dar, die im Fehring schwache Artikel mit restriktiven Relativsätzen erlauben. Keenan/Ebert (1973) stellen fest, dass der Kontrast zwischen opaken (nicht-spezifischen) und transparenten (spezifischen) Lesarten sich ebenfalls in der Wahl des Artikels niederschlägt, auch wenn ein restriktiver Relativsatz erscheint:

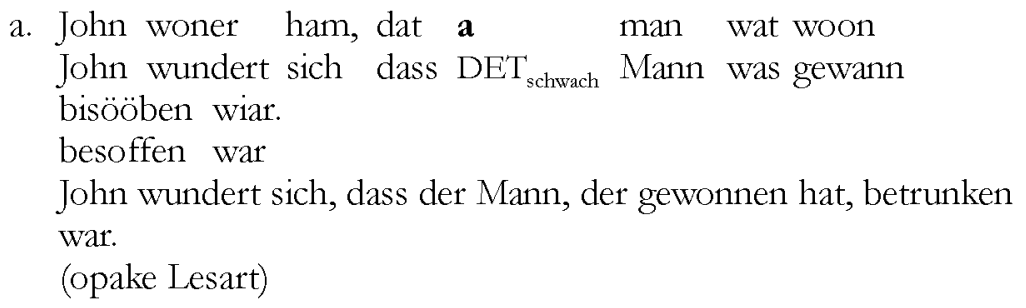

10 Ebert glossiert die Artikel mit d-Artikel und a-Artikel (in Anlehnung an die phonologische Form der Artikel im Fehring). Hier verwende ich $\mathrm{DET}_{\text {schrach }}$ für den a-Artikel und $\mathrm{DET}_{\text {stark }}$ für den d-Artikel. 
b. John woner ham, dat $\boldsymbol{*}_{\mathbf{a}} / \mathbf{d i}$ man wat

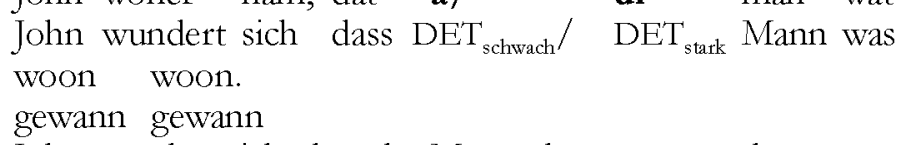

Für Verschmelzungsformen lässt sich ein vergleichbarer Effekt bei folgendem Beispiel erkennen:

(15) Was sie dazu getrieben hat, bleibt auch am Tag, an dem das ,Nein“ der grünen Insel zum Reformvertrag amtlich wird, im Nebulösen. (HAZ08/JUN.02578 Hannoversche Allgemeine, 14.06.2008, S. 3)

Wird hier die Verschmelzungsform am durch die Abfolge an dem ersetzt, wird der Tag als vorab erwähnt oder als fokalisiert dargestellt.

Die hier betrachteten Beispiele zeigen, dass restriktive Relativsätze unter spezifischen semantischen Umständen durchaus mit Verschmelzungsformen (und in anderen germanischen Sprachen mit den semantisch vergleichbaren schwachen definiten Artikeln) kombiniert werden können. Demnach ist die eingeschränkte Kombinierbarkeit von Verschmelzungsformen und restriktiven Relativsätzen als semantische Einschränkung einzustufen. Restriktive Relativsätze können somit als Evidenz für eine spezielle Syntax von Verschmelzungsformen im Deutschen ausgeschlossen werden.

\section{Verschmelzungen und Koordination: Französisch und Deutsch}

Wie Miller (1992) und Abeillé et al. (2003, 2006) zeigen, sind für die Verschmelzungsformen des Französischen Wechselwirkungen zwischen Morphologie und Syntax zu beobachten, da Verschmelzungsformen im Französischen gewisse Koordinationsmöglichkeiten ausschließen. Im Folgenden stelle ich zunächst die Evidenz dar, die belegt, dass französische Verschmelzungsformen zusätzliche Einschränkungen für die Syntax mit sich bringen. Anhand von Daten aus dem COSMAS II-Korpus soll anschließend belegt werden, dass solche Einschränkungen für die Verschmelzungsformen im Deutschen nicht zu beobachten sind: Verschmelzungsformen von Präposition und Artikel erlauben im Deutschen die gleichen Koordinationsmöglichkeiten wie volle Abfolgen von Präposition und Artikel.

\subsection{Französische Verschmelzungsformen und Koordination}

Miller (1992) weist als erster darauf hin, dass Verschmelzungsformen im Französischen nicht als lokale Verschmelzungen analysiert werden können 
und führt die folgenden Grammatikalitätsurteile als Beleg an (für de gelten die gleichen Urteile):

(16) J'ai parlé

ich-habe gesprochen
a. ̀̀ la
mère
et la
fille
A DET.FSG Mutter
und DET.FSG
Tochter
b. *au
père
et la
mère
A+DET.MSG Vater
und DET.FSG
Mutter
c. *à le
père
et la
mère
A DET.MSG Vater
und DET.FSG
Mutter
d. *à la
fille et le
A DET.FSG
Mädchen und
DET.MSG
garçon
e. à la
fille et l'autre
Junge
A DET.FSG Mädchen und DET.RED+andere Junge

„Ich habe mit a. der Mutter und der Tochter/b. dem Vater und der Mutter/c. dem Vater und der Mutter/d. dem Mädchen und dem Jungen/e. dem Mädchen und dem anderen Jungen gesprochen."

Beispiel (16a) belegt, dass die Koordination von zwei Nominalphrasen unter einer schwachen Präposition möglich ist. ${ }^{11}$ (16b) zeigt, dass auch für Sprecher, die (16a) akzeptieren, eine Kombination von Verschmelzungsform und N' nicht mit einer weiteren NP koordiniert werden kann. Auch in diesen Kontexten ist die Verschmelzung von Präposition und Artikel zwingend, wie (16c) zu entnehmen ist. Wie Miller (1992) betont, ist (16d) das frappierendste Beispiel, da hier eine potenzielle Verschmelzungsform, die bei Adjazenz von à und dem Artikel le im zweiten Konjunkt aufgetreten wäre, die Koordination unmöglich macht. Dieses Beispiel zeigt, dass Präposition und Artikel nicht adjazent sein müssen, um die Koordination zu blockieren: Es genügt, dass bei Adjazenz eine Verschmelzung auftreten würde. Verschmelzungen im Französischen beeinflussen somit die Syntax nicht nur lokal (wie in (16b)) sondern auch in Kontexten, die über lokale Adjazenz hinausgehen.

Das Beispiel (16e) zeigt schließlich, dass das Koordinationsproblem nicht auf das unterschiedliche Genus der NPs zurückgeführt werden kann: Wird die Möglichkeit einer Kontraktion durch élision des Artikels vor vokalisch anlautender NP ausgeschlossen (wie hier durch das pränominale Adjektiv autre ,anderer), wird die Koordination zweier NPs wieder möglich.

\footnotetext{
$11 \quad$ Ein Teil der französischsprachigen Muttersprachler lehnt eine Koordination von zwei NPs unter einer schwachen Präposition (d.h. à, de, en) grundsätzlich ab. Die von Miller (1992) angeführten Grammatikalitätsurteile treffen für Sprecher zu, die Koordinationen unter einer schwachen Präposition in Beispielen vom Typ (16a) zulassen.
} 


\subsection{Deutsche Verschmelzungsformen und Koordination}

Da französische Verschmelzungen Wechselwirkungen mit NP-Koordinationen zeigen, stellt sich nun die Frage, ob die Verschmelzungen des Deutschen ebenfalls die Koordinierbarkeit der Nominalphrasen in ihrem Komplement beeinflussen. Im Folgenden soll gezeigt werden, dass im Deutschen Verschmelzungsformen die Koordinierbarkeit nicht strukturell beeinträchtigen.

In gewissen Kontexten wird Koordination zweier Nominalphrasen unter einer Präposition als unglücklich empfunden. Der Duden (1984, S. 222, 2006, S. 624) empfiehlt beispielsweise, Verschmelzungsformen nicht mit zwei Nominalphrasen mit unterschiedlichen Artikeln zu kombinieren.

(17) a. Wir erkannten sie am Gang und ?der Haltung/an der Haltung.

b. Er berichtete über die Arbeit der Abgeordneten im Plenum und ?den Kommissionen/in den Kommissionen. (Duden 1984, S. 222)

Die Einschränkung, die diese Beispiele illustrieren, scheint dem französischen Beispiel (16b) zu entsprechen, in dem eine von einer Verschmelzungsform eingeleitete Präpositionalphrase keine Koordination mit einer weiteren NP zulässt. Die folgenden Beispiele belegen jedoch, dass diese Erklärung zu kurz greift.

(18) a. Als Spandau in den letzten fünf Minuten bereits im Gefühl des nahenden Sieges badete, kamen sie lediglich noch auf 8:10 heran, konnten aber am Jubel und der puren Wasserfreude der über 1000 Zuschauer nichts mehr ändern. (L98/AUG.08248 Berliner Morgenpost, 10.08.1998, S. 20)

b. Kapitalflucht und sinkende Spendenaufkommen auf privater Seite begleiten staatliche Kürzungen im Sozialhaushalt und der Entwicklungshilfe. (A98/FEB.11962 St. Galler Tagblatt, 26.02.1998)

c. Wer an diesem Tag Gefallen am Golf und der Übungsanlage findet, und sich entschließt, der Abteilung Golf beizutreten, spart die Aufnahmegebühr. (M06/MAI.38267 Mannheimer Morgen, 17.05.2006)

d. [...] Spötter wies darauf hin, dass die Beteiligung an manchen Veranstaltungen, zum Beispiel am Kirchgang und der Kranzniederlegung am Volkstrauertag, sehr schlecht gewesen sei. (BRZ07/ FEB.08539 Braunschweiger Zeitung, 01.02.2007)

e. Das Opfer, ihr 45-jähriger Freund, hatte bei der Messer-Attacke lebensgefährliche Verletzungen am Magen und der Bauchspeicheldrüse erlitten, aber dennoch selbst die Polizei verständigt. (M04/JAN.02210 Mannheimer Morgen, 12.01.2004)

f. Bisher pausierende Sänger und interessierte Damen und Herren, welche Freude am Chorgesang und der Kirchenmusik haben, sind uns herzlich willkommen. (M06/JAN.01947 Mannheimer Morgen, 10.01.2006) 
g. Vorrangig diskutiert werden müssten laut DRK unter anderem folgende Themenkomplexe: das Schaffen eines Rettungsdienstzweckverbandes, [...], einheitliche Entgelte im Rettungsdienstbereich sowie die Zusammenarbeit im Katastrophenschutz und dem Feuerwehrwesen. (BRZ06/FEB.12174 Braunschweiger Zeitung, 22.02.2006)

h. Die große Unterstützung für Livnis Konkurrenten Schaul Mofas, der offen für militärische Interventionen im Gazastreifen und dem Iran plädiert, belegt einen Rechtsruck innerhalb der Regierungspartei. (HMP08/SEP.01939 Hamburger Morgenpost, 19.09. 2008, S. 5)

In den Beispielen unter (18a-f) weisen die Nominalphrasen unterschiedliche Artikelformen auf, in Anlehnung an das Beispiel in (17). Meines Erachtens liegt jedoch kein grundlegender Kontrast zu den Beispielen in $(18 \mathrm{~g}-\mathrm{h})$ vor, in denen beide Nominalphrasen die gleiche Artikelform (M.DAT/N.DAT) erfordern. Möglicherweise werden die Koordinationen unter (17) und (18e/f) als weniger wohlgeformt empfunden, weil für diese Beispiele eine Koordination von bloßen Substantiven im Singular eine mögliche Alternative wäre:
a. an Gang und Haltung
b. Verletzungen an Magen und Bauchspeicheldrüse
(vgl. 17)
c. Freude an Chorgesang und Kirchenmusik

Die vorangehenden Beispiele kombinieren zwei Nominalphrasen im Singular. Wie die folgenden Beispiele mit einer zweiten Nominalphrase im Plural zeigen, sind auch unterschiedliche Numerusmerkmale in einer Koordination unter einer Verschmelzungsform zulässig:

(20) a. In den kommenden Tagen werden die Anleger die Entwicklung am Devisenmarkt und den internationalen Bondmärkten mit Aufmerksamkeit verfolgen. (A98/APR.26776 St. Galler Tagblatt, 28.04.1998)

b. Über 200 Kinder und zahlreiche Eltern sind im Laufe des Tages bei sonnigem Wetter hierher gekommen, um am Spieleparcours und den Führungen durch den Park teilzunehmen. (M01/AUG. 57951 Mannheimer Morgen, 07.08.2001)

c. Als neuen Pfeiler der Altersversorgung schlug er eine stärkere Beteiligung der Arbeitnehmer am Produktivkapital und den Gewinnen vor. (R97/MAR.17750 Frankfurter Rundschau, 07.03.1997)

In Cabredo Hofherr (im Erscheinen) habe ich vorgeschlagen, dass die Koordination unter einer Präposition durch semantische Faktoren beeinflusst wird. Die Koordination zweier Nominalphrasen unter einer Präposition wird beispielsweise begünstigt, wenn die Referenten der NPs als Teile eines komplexen Ganzen aufgefasst werden. Ein klares Beispiel für eine solche Konfi- 
guration bieten Koordinationen, in denen die zweite NP durch ein Possessivum eingeleitet wird, das auf die erste NP zurückverweist; in diesen Fällen wird die Verschmelzungsform bevorzugt:

(21) a. Erinnerungen ans Christkind und seine Gaben (I99/DEZ.53755 Tiroler Tageszeitung, 24.12.1999)

b. Organisationschef Herbert Gunz bedankte sich beim Direktor und seiner von Markus Dietrich geleiteten Spielcasino-Crew für das Engagement rund um die Mißwahl (...). (V97/FEB.08547 Vorarlberger Nachrichten, 14.02.1997)

Ein vergleichbarer semantischer Effekt lässt sich bei Infinitiven beobachten. Bei Infinitiven wird die Koordination unter einer gemeinsamen Verschmelzung bevorzugt, wenn die beschriebenen Tätigkeiten simultan ablaufen, während für Tätigkeiten, die zeitlich geordnet sind, die Verschmelzungsform wiederholt wird.

(22) a. Am Mittwochmorgen ging's ans Packen und Putzen. (A99/FEB. 08783 St. Galler Tagblatt, 06.02.1999)

b. Die Ukrainerin denkt nicht ans Heimkehren und ans Feiern. (A01/ AUG.20288 St. Galler Tagblatt, 08.08.2001)

Während Packen und Putgen in (22a) simultan ablaufen, sind Heimkehr und Feier in Beispiel (22b) zeitlich geordnet. Es muss betont werden, dass die Wiederholung der Verschmelzungsform in diesen Fällen auch von syntaktischen Faktoren abhängt: Wird einer der Infinitive durch ein Substantiv ersetzt, wird auch bei prinzipiell simultan ablaufenden Tätigkeiten die Verschmelzungsform wiederholt.

(23) Am nächsten Tag ging es ans Packen und an den grossen «Heimputz». (A98/JAN.06215 St. Galler Tagblatt, 31.01.1998)

Es soll nun weiter gezeigt werden, dass deutsche Verschmelzungsformen in allen Koordinationstypen auftreten können, in denen volle Abfolgen von Präposition und Artikel erscheinen können. Dies belegt, dass die deutschen Verschmelzungen die Koordinationsmöglichkeiten der Präpositionalphrase, die sie einleiten, nicht grundsätzlich syntaktisch einschränken.

Beispiel (24a) zeigt, dass Verschmelzungsformen untereinander koordiniert werden können. Verschmelzungsformen lassen des Weiteren auch eine Koordination mit einer Abfolge von vollen Formen zu (24b/c): ${ }^{12}$

Dies widerspricht der Darstellung in Hinrichs (1986). Laut Wiegand (2000, S. 67-68) unterscheiden sich Koordinationen mit und von Koordinationen mit anderen Konjunktionen: Mit und sollten die koordinierten Formen entweder einheitlich verschmolzen oder einheitlich unverschmolzen sein, während andere Konjunktionen die Koordination verschmolzener und unverschmolzener Abfolgen von Präposition und Artikel zulassen. Wie die Beispiele in (24) zeigen, sollte die Konjunktion und nicht als Ausnahme geführt werden. 
(24) a. Der Adventsmarkt am und im Schloss war für Groß und Klein gleichermaßen ein Erlebnis. (BRZ05/DEZ.05331 Braunschweiger Zeitung, 05.12.2005)

b. Veränderungen in der Siedlungsdichte von Kleinlebewesen im und auf dem Boden können einen Zusammenhang mit der Düngung haben. (M96/604.16489 Mannheimer Morgen, 13.04.1996)

c. Stadt und Land treten dafür ein, Arbeitsgruppen einzusetzen, um die Museums-Projekte auf dem und im Mönchsberg genau zu untersuchen. (N00/DEZ.57168 Salzburger Nachrichten, $09.12 .2000)$

Zwei Substantive mit ihren Komplementen können ebenfalls sowohl unter einer Abfolge von Präposition und Artikel (26a/b) als auch unter einer Verschmelzungsform $(25 \mathrm{a} / \mathrm{b})$ koordiniert werden.

(25) a. (..) im Geschrei und Gewühl von Menschenmassen (...)

b. Gewürdigt werden vor allem Perners langjähriges Engagement und ihre Arbeit zur Erforschung und Bekämpfung von Gewalt in der Familie und vor allem gegenüber Frauen. (N96/APR.16542 Salzburger Nachrichten, 19.04.1996)

(26) a. ... in dem immer unerträglicher werdenden Geschrei und Gewühl von Menschenmassen (...)

b. Er beschäftigt sich mit der Bekämpfung und Erforschung von Vorurteilen.

Die Beispiele in (18) und (20) belegen, dass die in Verschmelzungsformen enthaltene Präposition syntaktisch unabhängig ist und eine Koordination von Nominalphrasen als Komplement zulässt. Beispiel (27) zeigt weiter, dass auch der Artikel in Verschmelzungsformen für Koordinationen transparent sein muss.

(27) a. Lichtinstallation an und im Historischen Museum Speyer (M99/ NOV.72447 Mannheimer Morgen, 04.11.1999)

b. *Lichtinstallation an Historischen Museum Speyer

c. $\left[\mathrm{P}_{1}\right.$ und $\left.\mathrm{P}_{2}\right]+\mathrm{D}$

In diesem Beispiel muss der in der Verschmelzung im enthaltene Artikel für beide Präpositionen verfügbar sein, da das Beispiel (27b) ohne Artikel ungrammatisch ist. Deutsche Verschmelzungsformen verfügen also über die gleichen Koordinationsmöglichkeiten wie unverschmolzene Abfolgen von Präposition und Artikel.

Wie in der Diskussion um das Beispiel (16d) dargestellt, macht Miller (1992) die Beobachtung, dass für das Französische poten:ielle Verschmelzungen Koordinationen blockieren können. Auch diese Einschränkung trifft für das Deutsche nicht zu: Präpositionen sowie Nominalphrasen können auch dann koordiniert werden, wenn die einleitende Präposition unter Adjazenz mit dem Artikel eine Verschmelzungsform bilden würde (28a/b). 
(28) a. Dabei stießen sie im Laufe der Zeit auf] eine wundersame Tier- und Pflanzenwelt in und auf dem Dom (N97/APR.16459 Salzburger Nachrichten, 21.04.1997)

b. eine [...] Tier- und Pflanzenwelt im Dom

Abschließend können wir also festhalten, dass sich Verschmelzungen im Deutschen für Koordinationen syntaktisch wie eine Abfolge nicht-reduzierter Formen verhalten.

\section{Analyse: Syntaktisch konditionierte Allomorphie}

Sowohl für das Französische als auch für das Deutsche ist eine Analyse der Verschmelzungsformen als phonologische Kontraktion nicht überzeugend. In beiden Sprachen kann die Verschmelzung nicht für alle Abfolgen eintreten, die mit der Abfolge von Präposition und Artikel homophon sind. Im Deutschen ist die Verschmelzungsform für Abfolgen von Präposition und Relativpronomen unmöglich (Hinrichs 1986), im Französischen können die Verschmelzungsformen Abfolgen von Präposition und pronominalen Klitika nicht ersetzen (Abeillé et al. 2003).

(29) a. Das Haus, in dem/*im Fritz wohnt, wird verkauft. (Relativpronomen)

b. Jean a peur Jean hat Angst

i. de le/ $*$ du mettre.

von pron.ACC3MSG DU anziehen

ii. *de le/ du maitre

von DET.MSG/ DE+DET.MSG Meister.

Jean hat Angst, (i) ihn anzuziehen/(ii) vor dem Meister.

Hier möchte ich vorschlagen, dass Verschmelzungsformen im Deutschen post-syntaktisch über zwei syntaktische Endstellen eingefügt werden (siehe ,lexical sharing' in Wescoat 2002). Die Verschmelzungsformen unterscheiden sich jedoch klar von einfacher Klitisierung, da die Form suppletiv ist (siehe Raffelsiefen 1987) und demzufolge in einer post-syntaktischen Komponente gespeichert sein muss.

Die Einfügung der Verschmelzungsformen unterliegt außerdem syntaktischen Bedingungen: Die Präposition verschmilzt nur mit dem Artikel ihres Komplements (siehe van Riemsdijk 1998). Die hier vorgeschlagene Analyse behandelt somit Verschmelzungsformen als post-syntaktische Klitisierung, deren morphologische Realisierung durch eine Allomorphieregel mit syntaktischen Anwendungsbedingungen bestimmt wird. 
6.1 Die Verschmelzungsformen werden post-syntaktisch eingefügt

In der Diskussion der Koordinationsdaten habe ich gezeigt, dass sich Verschmelzungsformen syntaktisch transparent verhalten: Sowohl die Präposition als auch der enthaltene Artikel sind für Koordinationen getrennt verfügbar. Hier folge ich dem Vorschlag in Wescoat (2002), dass lexikalische Einfügung post-syntaktisch über mehrere adjazente syntaktische Endstellen möglich ist (für eine ähnliche Analyse siehe Zwicky 1987). Verschmelzungsformen müssen in einer post-syntaktischen Komponente eingeführt werden, da nur nach Aufbau der syntaktischen Struktur erkennbar ist, ob Präposition und semantisch schwach definiter Artikel adjazent sind und durch eine Verschmelzung lexikalisch realisiert werden können.

\subsection{Die Verschmelzungsformen sind suppletiv}

Trotz der Tatsache, dass deutsche Verschmelzungen sich syntaktisch wie zwei Einheiten verhalten, können die Verschmelzungsformen nicht als einfache Klitisierung (siehe Zwicky 1977) angesehen werden, da die Form der Verschmelzungsformen suppletiv ist (siehe Raffelsiefen 1987). So würde bei der einfachen Klitisierung von an mit einem reduzierten Artikel ' $m$ die Form mit einem Schwa in (30b) erwartet, nicht die tatsächliche Form in (30a):
a. an + ' $m-\rightarrow$ am (Verschmelzung)
b. an+'m- $\rightarrow$
[anəm] (phonologisch erwartete Form)

Folglich müssen Verschmelzungsformen mit ihrer suppletiven Form im Lexikon gespeichert sein (vergleichbare Belege für suppletive Verschmelzungsformen im Ruhrdeutschen finden sich in Schiering 2002, S. 39, 2005).

\subsection{Die Verschmelzungsformen sind syntaktisch konditioniert}

In den von Wescoat (2002) analysierten Fällen von ,lexical sharing findet die Einfügung der Verschmelzungsformen bei Adjazenz der relevanten syntaktischen Positionen automatisch statt. Dieser Ansatz kann so für die Verschmelzungsformen des Deutschen nicht übernommen werden (pace Wescoat 2007), da Adjazenz von Präposition und Artikel nicht ausreicht, um eine Verschmelzungsform zu legitimieren. Wie van Riemsdijk (1998, S. 655) zeigt, kann die Verschmelzung nur in Kontexten auftreten, in denen der Artikel das Komplement der Präpositionalphrase einleitet. Strukturell tiefer eingebettete Artikel können auch bei linearer Adjazenz nicht mit der Präposition verschmelzen:
a. von [dem König treu ergebenen] Dienern
b. *vom König treu ergebenen Dienern (van Riemsdijk 1998, S. 655) 
Die hier vertretene Analyse behandelt Verschmelzungsformen als syntaktisch konditionierte Allomorphie, die als Gegenstück zu allgemein bekannten Fällen phonologisch konditionierter Allomorphie (32) zu verstehen ist. Als das paradigmatische Beispiel einer phonologisch konditionierten Allomorphie kann die Allomorphie des englischen unbestimmten Artikels a/ an gelten:

(32) Allomorphie des englischen unbestimmten Artikels
a. a vor Konsonanten a pear ,eine Birne
b. an vor Vokalen an apple, ein Apfel

\subsection{Alternative Lösungsansätze}

Ich möchte im Folgenden kurz begründen, warum ich den in der Literatur vorgeschlagenen Analyseansätzen in der hier vertretenen Analyse für die deutschen Verschmelzungsformen nicht folge. Im Wesentlichen behandeln die Ansätze von Abeillé et al. (2003, 2006) und van Riemsdijk (1998) Verschmelzungsformen als flektierte Präpositionen, wobei Flexion als lexikalischer oder aber als syntaktischer Prozess (Kopfbewegung) aufgefasst wird.

Abeillé et al. (2003, 2006) schlagen eine lexikalische Analyse vor, derzufolge Verschmelzungsformen als flektierte Präpositionen aufgefasst werden, die im Lexikon als Lexeme gespeichert sind. Diese Autoren nehmen an, dass die französischen Verschmelzungsformen sich durch die syntaktische Selektion einer reduzierten Nominalphrase $(\mathrm{N})$ auszeichnen, oder anders formuliert, dass die Komplemente von Verschmelzungsformen die Form einer DP ohne D-Kopf haben. Als Evidenz für diese Analyse führen Abeillé et al. die eingeschränkten Koordinationsmöglichkeiten unter Verschmelzungsformen an: Beispiele wie (16b) sind demzufolge ungrammatisch, weil hier eine PP mit einer DP koordiniert wird. Für die deutschen Verschmelzungsformen ist dieses Argument nicht einschlägig, da vergleichbare Koordinationen im Deutschen möglich sind.

Van Riemsdijk (1998) analysiert Verschmelzungsformen als Kopfbewegung des Artikels an die Präposition. Dieser Vorschlag kann als eine syntaktische Version der Analyse als flektierter Präposition interpretiert werden, da in dem zugrundeliegenden Modell Flexion des Verbes beispielsweise ebenfalls als Kopfbewegung analysiert wird. Bei diesem Ansatz bleiben meines Erachtens verschiedene Fragen offen. Erstens scheint es keinen plausiblen Auslöser für die Kopfbewegung zu geben: Sowohl Präpositionen als auch schwache Artikel treten als alleinstehende Einheiten auf.

a. semantisch schwache Artikel treten ohne Präposition auf:

der Dom, der Bodensee, die Zugspitze

b. Präpositionen treten ohne Artikel auf:

an Land, auf Wanderschaft

Zweitens können Präpositionen über einer DP koordiniert werden, auch wenn die zweite Präposition die Form einer Verschmelzungsform hat: 
an und im Historischen Museum Speyer

Die Interpretation des Beispiels (35) legt nahe, dass es sich bei Koordinationen von Präpositionen um eine einzige Nominalphrase handelt, da kein Multiplikationseffekt eintritt: In (35) handelt es sich um zwei Kirchen, nicht um vier. ${ }^{13}$

an und auf zwei Kirchen

In dieser Konfiguration scheint eine Kopfbewegung unplausibel, da in vergleichbaren Beispielen mit flektierten Verben die Flexion unbedingt an beiden Verben realisiert werden muss:

$$
\text { spielte und tanzte nicht: } * \text { [spiel- und tanz]-te }
$$

Im Gegensatz zu den Flexionsanalysen in Abeillé et al. $(2003,2006)$ und van Riemsdijk (1998), schlägt Embick (2007b) eine Analyse der französischen Verschmelzungsformen als post-syntaktische Bewegung vor. Diese Analyse stützt sich auf ein morphologisches Modell, das post-syntaktische Bewegungen in der morphologischen Komponente zulässt. In dem hier vertretenen Ansatz bleiben Bewegungen der syntaktischen Komponente vorbehalten: Die Verschmelzungsformen werden als Spezialfall der lexikalischen Einfügung behandelt, in der zwei syntaktische Endstellen gleichzeitig abgedeckt werden.

\section{Fazit}

Im vorliegenden Beitrag habe ich eine Analyse der Verschmelzungsformen von Präposition und Artikel im Deutschen als post-syntaktische Allomorphie vorgeschlagen, die zusätzlich syntaktischen Anwendungsbedingungen unterliegt.

Die Annahme einer post-syntaktische Einfügung der Verschmelzungsformen stützt sich auf die Beobachtung, dass sowohl Präposition als auch Artikel in Verschmelzungsformen für die Syntax zugänglich sind. Wie gezeigt wurde, haben Verschmelzungsformen in Relativsätzen und in Koordinationen die gleichen syntaktischen Kombinationsmöglichkeiten wie nicht-reduzierte Abfolgen von Präposition und Artikel. Deutsche Verschmelzungsformen unterscheiden sich damit von den Verschmelzungsformen des Französischen, die die Koordinierbarkeit von Nominalphrasen beeinträchtigen.

13 Diese Argumentation lehnt sich an Abbott (1976) an, die zeigt, dass V-Koordination nicht mit Objekt-Ellipse gleichgestellt werden kann, da die Konstruktionen verschiedene Interpretationen haben. Während in (ia) Hobbs 16 Melodien summt oder pfeift, können es in (ib) bis zu 32 Melodien sein.

(i) a. Hobbs whistled and hummed a total of 16 tunes.

b. Hobbs whistled a total of 16 tunes and hummed a total of 16 tunes. 


\section{Literatur}

Abbott, Barbara (1976): Right node raising as a test for constituenthood. In: Linguistic Inquiry 7, S. 639-642.

Abeillé, Anne et al. (2003): The syntax of French à and de: an HPSG analysis. In: Proceedings of the ACL-SIGSEM Workshop on the Linguistic Dimensions of Prepositions. Toulouse, S. 133-144.

Abeillé, Anne et al. (2006): The syntax of French à and de: an HPSG analysis. In: SaintDizier, Patrick ( $\mathrm{Hg}$ ): Syntax and semantics of prepositions. (= Text, Speech and Language Technology 29). Dordrecht.

Ackema, Peter/Neeleman, Ad (2004): Beyond morphology: interface conditions on word formation. (= Oxford Studies in Theoretical Linguistics 6). Oxford u.a.

Ackema, Peter/Neeleman, Ad (2007): Morphology $\neq$ syntax. In: Ramchand/Reiss (Hg), S. 325-352

Börjars, Kersti/Vincent, Nigel/Chapman, Carol (1997): Paradigms, periphrases and pronominal inflection: a feature-based account. In: Booij, Geert/van Marle, Jaap (Hg.): Yearbook of Morphology 1996. Dordrecht, S. 155-180.

Bresnan, Joan (2001): Explaining morphosyntactic competition. In: Baltin, Mark/Collins, Chris ( $\mathrm{Hg}$.): Handbook of Contemporary Syntactic Theory. Oxford, S. 1-44.

Breu, Walter (2004): Der definite Artikel in der obersorbischen Umgangssprache. In: Krause, Marion/Sappok, Christian (Hg): Slavistische Linguistik 2002. Referate des XXVIII. Konstanzer Slavistischen Arbeitstreffens, Bochum, 10.-12.9.2002. (= Slavistische Beiträge 434 ). München, S. 9-57.

Cabredo Hofherr, Patricia (i.Ersch.) Preposition-determiner amalgams in German and French at the syntax-morphology interface. In: Ackema, Peter et al. (Hg.): Comparative Germanic syntax: the state of the art. Amsterdam/Philadelphia.

Chumakina, Marina/Corbett, Greville G. (Hg.) (i.Ersch.): Periphrasis: The role of syntax and morphology in paradigms. (= Proceedings of the British Academy). British Academy \& Oxford University Press.

Duden (1984): Der Duden in zehn Bänden. Bd. 4: Grammatik der deutschen Gegenwartssprache. Hrsg. u. bearb. v. Günter Drosdowski. 4., völlig neu bearb. u. erw. Aufl. Mannheim u.a.

Duden (2006): Der Duden in zwölf Bänden. Bd. 4: Die Grammatik. Hrsg. v. d. Dudenredaktion. Neudr. d. 7., völlig neu erarb. u. erw. Aufl. Mannheim u.a.

Ebert, Karen H. (1971a): Referenz, Sprechsituation und die bestimmten Artikel in einem nordfriesischen Dialekt (Fehring). (= Studien und Materialien/Nordfriisk Instituut 4). Bräist/Bredstedt.

Ebert, Karen H. (1971b): Zwei Formen des bestimmten Artikels. In: Wunderlich, Dieter $(\mathrm{Hg}$ ): Probleme und Fortschritte der Transformationsgrammatik. Referate des 4. Linguistischen Kolloquiums, Berlin 6.-10. Oktober 1969. (= Linguistische Reihe 8). München, S. 159-173.

Embick, David (2007a): Blocking effects and analytic/synthetic alternations. In: Natural Language and Linguistic Theory 25, S. 1-37. 
Embick, David (2007b): Linearization and local dislocation: derivational mechanisms and interactions. In: Linguistic Analysis 33, S. 303-336.

Embick, David/Noyer, Rolf (2001): Movement operations after syntax. In: Linguistic Inquiry 32, S. 555-595.

Embick, David/Noyer, Rolf (2007): The syntax/morphology interface. In: Ramchand/ Reiss (Hg:), S. 289-324.

Hankamer, Jorge/Mikkelsen, Line (2005): When movement must be blocked: a reply to Embick and Noyer. In: Linguistic Inquiry 36, S. 85-125.

Hartmann, Dietrich (1978): Verschmelzungen als Varianten des bestimmten Artikels? In: Hartmann, Dietrich/Linke, Hansjürgen/Ludwig, Otto (Hg.): Sprache in Gegenwart und Geschichte. Festschrift für Heinrich Matthias Heinrichs zum 65. Geburtstag. Wien, S. 68-81.

Hartmann, Dietrich (1980): Über Verschmelzungen von Präposition und bestimmtem Artikel. In: Zeitschrift für Dialektologie und Linguistik 47, S. 160-183.

Hartmann, Dietrich (1982): Deixis and anaphora in German dialects: the semantics and pragmatics of two definite articles in dialectical varieties. In: Weissenborn, Jürgen/ Klein, Wolfgang $(\mathrm{Hg})$ : Here and there: cross-linguistics studies on deixis and demonstration. Amsterdam/Philadelphia, S. 187-207.

Heinrich, Heinrich Matthias (1954): Studien zum bestimmten Artikel in den germanischen Sprachen. (= Beiträge zur deutschen Philologie 1). Giessen.

Hinrichs, Erhard W. (1986): Verschmelzungsformen in German - a GPSG analysis. In: Linguistics 24, S. 939-955.

Keenan, Ed/Ebert, Karen (1973): A note on marking transparency and opacity. In: Linguistic Inquiry 4, S. 421-424.

Löbner, Sebastian (1985): Definites. In: Journal of Semantics 4, S. 279-326.

Miller, Philip (1992): Clitics and constituents in Phrase Structure Grammar. New York.

Miller, Philip/Pullum, Geoffrey K./Zwicky, Arnold (1997): The principle of phonologyfree syntax: four apparent counterexamples in French. In: Journal of Linguistics 33, S. $67-90$.

Nübling, Damaris (2005): Von in die über $i n$ 'n und $i n s$ bis im: Die Klitisierung von Präpositionen und Artikel als „Grammatikbaustelle“. In: Leuschner, Torsten/Mortelmans, Tanja/de Groodt, Sarah (Hg.): Grammatikalisierung im Deutschen. Berlin, S. $105-131$.

Poser, William (1992): Blocking of phrasal constructions by lexical items. In: Sag, Ivan A./Szabolcsi, Anna (Hg.): Lexical matters. (= CSLI Lecture Notes 24). Stanford, S. 111-130.

Puig-Waldmüller, Estela (2008): Contracted preposition-determiner forms in German: semantics and pragmatics. Diss., Universitat Pompeu Fabra. Barcelona.

Raffelsiefen, Renate (1987): „Verschmelzungsformen“ in German: a lexical analysis. In: Linguistic Analysis 17, S. 123-146.

Ramchand, Gillian/Reiss, Charles (Hg.) (2007): The Oxford Handbook of Linguistic Interfaces. Oxford. 
Riemsdijk, Henk van (1998): Head-movement and adjacency. In: Natural Language and Linguistic Theory 16, S. 633-678.

Schiering, René (2002): Klitisierung von Pronomina und Artikelformen. Eine empirische Untersuchung am Beispiel des Ruhrdeutschen. (= Institut für Sprachwissenschaft, Universität zu Köln, Arbeitspapiere (Neue Folge) 44). Köln.

Schiering, René (2005): Flektierte Präpositionen im Deutschen? Neue Evidenz aus dem Ruhrgebiet. In: Zeitschrift für Dialektologie und Linguistik 72, S. 52-79.

Schwarz, Florian (2009): Two types of definites in natural language. Diss., Universität von Massachusetts. Amherst.

Stewart, Thomas/Stump, Gregory (2007): Paradigm function morphology and the morphology/syntax interface. In: Ramchand/Reiss (Hg.), S. 383-422.

Studler, Rebekka (2008): Artikelparadigmen. Form, Funktion und syntaktisch-semantische Analyse von definiten Determinierern im Schweizerdeutschen. Dissertationsmanuskript. Zürich/Konstanz.

Wescoat, Michael (2002): On lexical sharing. Diss., Universität Stanford. Stanford, CA.

Wescoat, Michael (2007): Preposition-determiner contractions: an analysis in optimality-theoretic lexical-functional grammar with lexical sharing. In: Butt, Miriam/ King, Tracy Holloway $(\mathrm{Hg}$ ): Proceedings of the LFG07 Conference. Stanford, CA, S. $439-459$.

Wespel, Johannes (2008): Descriptions and their domains. Patterns of definiteness marking in French-related creoles. Diss. Stuttgart. Internet: http://elib.uni-stuttgart.de/opus/ volltexte/2008/3654/pdf/Wespel_Diss_OPUS.pdf (Stand: 25.08.2011).

Wiegand, Herbert Ernst (2000): Verschmelzungen in allgemeinen einsprachigen Wörterbüchern des Deutschen. In: Kramer, Undine (Hg): Lexikologisch-lexikographische Aspekte der deutschen Gegenwartssprache. Symposiumsvorträge, Berlin 1997. (= Lexicographica, Series maior 101). Tübingen, S. 59-96.

Zwicky, Arnold (1977): On clitics. Ms. Bloomington, IN. Internet: www.stanford.edu/ Zwicky/on_clitics.pdf (Stand: 25.08.2011).

Zwicky, Arnold (1987): French prepositions: no peeking. In: Phonology Yearbook 4, S. 211-227.

Zwicky, Arnold/Pullum, Geoffrey K. (1986): The principle of phonology-free syntax: introductory remarks. In: Ohio State University Working Papers in Linguistics 32. Columbus, OH, S. 63-91. 